\title{
Abnormal Sensory Protein Expression and Urothelial Dysfunction in Ketamine-Related Cystitis in Humans
}

\author{
Yao Chou Tsai ${ }^{1,2}$, Lori Birder ${ }^{3}$, Hann-Chorng $\mathrm{Kuo}^{2,4}$ \\ ${ }^{1}$ Division of Urology, Department of Surgery, Taipei Tzu Chi General Hospital, Buddhist Tzu Chi Medical Foundation, Taipei, Taiwan \\ ${ }^{2}$ Department of Urology, School of Medicine, Tzu Chi University, Hualien, Taiwan \\ ${ }^{3}$ Department of Medicine and Pharmacology, University of Pittsburgh School of Medicine, Pittsburgh, PA, USA \\ ${ }^{4}$ Department of Urology, Buddhist Tzu Chi General Hospital, Hualien, Taiwan
}

Purpose: The aim of this study was to analyze patterns of sensory protein expression and urothelial dysfunction in ketaminerelated cystitis $(\mathrm{KC})$ in humans.

Methods: Biopsies of bladder mucosa were performed in $29 \mathrm{KC}$ patients during cystoscopy. Then specimens were analyzed for tryptase, zonula occludens-1 (ZO-1), E-cadherin, and terminal deoxynucleotidyl transferase-mediated dUTP nick end-labeling (TUNEL) with immunofluorescence staining and quantification. In addition, 10 healthy control bladder specimens were analyzed and compared with the KC specimens. Another 16 whole bladder specimens obtained from partial cystectomy were also analyzed for the muscarinic receptors $\mathrm{M} 2$ and $\mathrm{M} 3$, endothelial nitric oxide synthase (eNOS), inducible nitric oxide synthase (iNOS), $\beta-3$ adrenergic receptors ( $\beta 3$-ARs), and the $\mathrm{P}_{2} \mathrm{X}_{3}$ receptor by western blotting. In addition, 3 normal control bladder specimens were analyzed and compared with the KC specimens.

Results: The KC bladder mucosa revealed significantly less expression of ZO-1 and E-cadherin, and greater expression of TUNEL and tryptase activity than the control samples. The expression of $M 3$ and $\beta 3-\mathrm{AR}$ in the $\mathrm{KC}$ specimens was significantly greater than in the controls. The expression of iNOS, eNOS, M2, and P2X $\mathrm{X}_{3}$ was not significantly different between the KC and control specimens.

Conclusions: The bladder tissue of KC patients revealed significant urothelial dysfunction, which was associated with mastcell mediated inflammation, increased urothelial cell apoptosis, and increased expression of the M3 and $\beta 3-\mathrm{AR}$.

Keywords: Ketamine; Urothelium

- Research Ethics: This study was approved by the Institutional Review Board of the Tzu Chi Hospital (approval number: TCGH IRB 102-78).

- Conflict of Interest: No potential conflict of interest relevant to this article was reported.

\section{INTRODUCTION}

Ketamine, an antagonist of the N-methyl-D-aspartic acid receptor complex, has been used since the 1960s as an anesthetic or analgesic [1]. It remains the most commonly used anesthetic in veterinary medicine, and is also used in children and for the management of chronic pain. The psychosis-like symptoms induced by ketamine have led to its use as a pharmacological model of schizophrenia [2]. These effects also have led to the recreational use of ketamine. Due to its easy availability, ketamine has been the most common illicit drug of abuse reported by the Food and Drug Administration in Taiwan since 2006.

Corresponding author: Hann-Chorng Kuo (iD http://orcid.org/0000-0001-7165-4771 Department of Urology, Buddhist Tzu Chi General Hospital, Hualien, 707, section 3, Chung Yang Road, Hualien, Taiwan

E-mail: hck@tzuchi.com.tw / Tel: +886-0970333975 / Fax +886-266289009

Submitted: May 12, 2016 / Accepted after revision: June 23, 2016 
The clinical presentation of ketamine-related cystitis (KC) includes remarkable dysuria, urinary frequency and/or urgency, urge incontinence, and bladder pain. KC shares many common histopathological features with interstitial cystitis/bladder pain syndrome (IC/BPS), including urothelial ulceration, inflammatory cell infiltration, and varying degrees of bladder wall fibrosis [3]. However, the degree of bladder wall inflammation and fibrosis are more severe in $\mathrm{KC}$, which results in a contracted bladder, hydronephrosis, and even upper urinary tract damage [4]. However, the exact etiopathological mechanism of the development of KC remains unknown.

Findings from previous animal studies support the hypothesis that urothelial dysfunction and abnormal sensory protein expression may play a role in the pathogenesis of $\mathrm{KC}$ [5]. A recent human study also revealed augmented inflammatory protein and sensory protein expression in KC bladder biopsy samples [6]. The goal of the present study was to use human bladder biopsies and whole bladder specimens to investigate the effect of $\mathrm{KC}$ on proteins involved in urothelial barrier and sensory functions.

\section{MATERIALS AND METHODS}

\section{Patients}

Patients who had been admitted to the Hualien Tzu Chi General Hospital from 2011 to 2014 with a history of the recreational abuse of ketamine for not less than 6 months were retrospectively recruited for this study. This study was approved by the Institutional Review Board of the Tzu Chi Hospital (approval number: TCGH IRB 102-78). The frequency of ketamine use in these patients was not less than twice per week, and the dosage of ketamine was not less than $3 \mathrm{~g}$ each time. All of the included patients reported lower urinary tract symptoms including urgency, severe frequency, and intolerable bladder pain with a duration of at least 3 months. The severity of bladder discomfort was measured using a visual analog pain scale (0-10). The exclusion criteria were patients with a history of asthma, collagen vascular diseases, neoplasms, or parasitic infection, and patients with concomitant acute bacterial cystitis, stress urinary incontinence, urolithiasis, or neurogenic voiding dysfunction. The control sample of healthy biopsy specimens was obtained from patients with stress urinary incontinence treated over the same period of time (part 1 of the study) and the whole-bladder control specimens were obtained from patients who underwent radical cystectomy over the same period of time (part 2 of the study).

\section{Sample Collection}

The patients' medical histories were comprehensively reviewed, including the duration of ketamine usage. Patients using ketamine before hospitalization were considered active drug users. All enrolled patients were hospitalized for diagnostic cystoscopic hydrodistention under general anesthesia with an intravesical pressure of $80 \mathrm{~cm} \mathrm{H}_{2} \mathrm{O}$. At the end of cystoscopy, random biopsies of the bladder specimens were taken at 2 sites on the bladder base and posterior wall approximately $2 \mathrm{~cm}$ above the ureteral orifice. Every specimen included only the mucosa and was approximately $2 \mathrm{~mm}$ thick. The specimens were sent for histological examination by a single pathologist who was blinded to the diagnosis of the patient. Some specimens were also embedded in optimum cutting temperature (OCT) medium and stored frozen at $-80^{\circ} \mathrm{C}$ for further investigation. If the patients had persistent bladder pain, severe frequency symptoms, and a small cystometric bladder capacity of less than 150 $\mathrm{mL}$, conservative management or augmentation enterocystoplasty was recommended for the definitive treatment [7].

\section{Immunhistochemical Staining (Part 1 of the Study)}

Bladder biopsies taken during the cystoscopic hydrodistention procedure were investigated to determine the expression levels of mast cell tryptase, zonula occludens-1 (ZO-1), E-cadherin, terminal deoxynucleotidyl transferase-mediated dUTP nick end-labeling (TUNEL), and transforming growth factor (TGF)- $\beta$. After biopsy, the bladder specimen was initially fixed in ice-cold $4 \%$ formaldehyde phosphate-buffered saline $(\mathrm{pH}$, 7.4) solution for 1 hour. The specimens were then rinsed with phosphate-buffered saline containing $15 \%$ sucrose at $4^{\circ} \mathrm{C}$ overnight. The specimens were embedded in OCT medium (Miles, Elkhart, IN, USA) and stored at $-80^{\circ} \mathrm{C}$. Four alternate sections per specimen were cryosectioned with a thickness of $8 \mu \mathrm{m}$ and were placed on Polysine glass slides (Thermo Fisher Scientific, Waltham, MA, USA). The sections were then fixed in acetone at $-20^{\circ} \mathrm{C}$ and incubated with blocking solution (BioGenex Laboratories, Santa Cruz, CA, USA). Subsequently, they were incubated overnight at $4^{\circ} \mathrm{C}$ with the following primary antibodies: antihuman E-cadherin (BD Biosciences, Franklin, NJ, USA), antihuman ZO-1 (Invitrogen, Burlington, ON, Canada), or antihuman mast cell tryptase (Chemicon, Temecula, CA, USA). After rinsing the sections with $0.1 \%$ Tween 20 (Sigma Aldrich, St. Louis, MO, USA) in phosphate-buffered saline, rabbit antimouse immunoglobulin/fluorescein isothiocyanate or swine anti-rabbit immunoglobulin/fluorescein isothiocyanate (Dako- 
Cytomation Denmark, Copenhagen, Denmark) were used on the sections, which were then incubated for 1 hour at room temperature. The sections were then counterstained with 4,6-diamidino-2-phenylindole (Sigma Aldrich) and the negative controls were processed using the same procedure, except for the primary antibodies. Immunofluorescence-stained images of mast cell tryptase and E-cadherin were assessed using fluorescence microscopy and then processed with a digital imaging system (Carl Zeiss, Oberkochen, Germany). The intensity and distribution of ZO-1 were characterized using a confocal microscope (Carl Zeiss). The expression levels of ZO-1 and Ecadherin in the urothelium were quantified using the Image J program, developed by the National Institutes of Health (Bethesda, MD, USA). The number of tryptase-positive mast cells was counted in 5 consecutive high-power fields $(\times 400)$ and the percentage was calculated as the number of tryptasepositive mast cells divided by the total cells per unit area (4 $\left.\mu \mathrm{m}^{2}\right) \times 100$. Three tissue sections per sample from the KC bladder samples and controls were quantified, and the mean values were used for subsequent statistical analyses.

\section{TUNEL Assay (Part 1 of the Study)}

The specimens were immersed in an ice-cold solution of $4 \%$ formaldehyde in phosphate-buffered saline $(\mathrm{pH}, 7.4)$ for 1 hour and then rinsed with ice-cold phosphate-buffered saline containing 15\% sucrose for 12 hours. The specimen was then embedded in OCT medium (Miles, Elkhart, IN, USA) and stored at $-80^{\circ} \mathrm{C}$. All tissues were sectioned $(6 \mu \mathrm{m})$ and collected onto new silane III--coated slides, then incubated with 100-120 $\mu \mathrm{g} /$ $\mathrm{mL}$ of proteinase K (Calbiochem, Dormstadt, Germany) at room temperature for 10 minutes, and washed with phosphatebuffered saline. The section was covered with $100 \mu \mathrm{L}$ of terminal deoxynucleotidyl transferase (TdT) equilibration buffer (Calbiochem), and then incubated at room temperature for 30 minutes. After carefully blotting the $1 \times \mathrm{TdT}$ equilibration buffer from the specimens, the TdT labeling reaction mixture (Calbiochem) was used on the specimens, which were then incubated for 90 minutes at $37^{\circ} \mathrm{C}$. The positive controls, apoptotic HL-60 cells (Calbiochem), were treated with $1-\mu \mathrm{g} / \mu \mathrm{L}$ DNase I in Tris-buffered saline/ $1 \mathrm{mM} \mathrm{MgSO}_{4}$ (Promega Corp., Madison, WI, USA) at room temperature for 20 minutes, followed by the negative controls generated by substituting $\mathrm{dH}_{2} \mathrm{O}$ for the $\mathrm{TdT}$ enzyme in the reaction mixture. After washing with phosphatebuffered saline, the sections were mounted using FluoresceinFragELTM mounting medium (Calbiochem), and the total cell population was evaluated with a 330- to $380-\mathrm{nm}$ filter for 4 ', 6-diamidino-2-phenylindole, while the labeled nuclei were evaluated with a standard fluorescein filter (465-495 nm).

\section{Western Blotting (Part 2 of the Study)}

Another 16 bladder specimens obtained from partial cystectomy were analyzed for the expression of muscarinic receptors (M2 and M3), TGF- $\beta 3$, endothelial nitric oxide synthase (eNOS), inducible nitric oxide synthase (iNOS), $\beta$ - 3 adrenoceptor ( $\beta 3-\mathrm{AR})$, and P2X3 using Western blotting. Initially, collected human bladder tissue samples were homogenized with liquid nitrogen, and then the protein was separated using a lysis buffer including a protease inhibitor cocktail and a phosphatase inhibitor cocktail (Roche Diagnostics, Mannheim, Germany) for 60 minutes. Soluble proteins were quantified using the BioRad Protein Assay Dye Reagent Concentrate (Bio-Rad Laboratories Inc., Hercules, CA, USA) and added (10 $\mu \mathrm{g})$ to $8 \%-16 \%$ PreciseTM Tris-glycine gel (Thermo Scientific, Scoresby, Australia). After gel electrophoresis, transfer to the $0.2-\mu \mathrm{m}$ polyvinylidene difluoride membrane was carried out using 3\% skim milk as a blocking buffer for 1 hour, followed by the primary antibodies for $\mathrm{P}_{2} \mathrm{X}_{3}$ (GeneTex Inc., Irvine, CA, USA), M2 (GeneTex Inc.), M3 (GeneTex Inc.), eNOS (GeneTex Inc.), iNOS (Abcam, Cambridge, UK), $\beta 3$-AR (Abcam), or GAPDH (Cell Signaling Technology Danvers, MA, USA) as a positive control. The sample was then incubated overnight at $4^{\circ} \mathrm{C}$, washed with Tris-buffered saline and Tween 20, and incubated with the secondary antibody (goat anti-rabbit IgG-HRP, 1:3,000, Santa Cruz Biotechnology, Santa Cruz, CA, USA), Membranes were probed with an enhanced chemiluminescence reagent (ECL; Millipore Corp., Billerica, MA, USA) and exposed to X-ray film.

\section{Statistical Analysis}

Continuous variables were analyzed as the mean \pm standard deviation and were tested for normality using the Shapiro-Wilk test. The Mann-Whitney U-test or the independent-samples ttest was used for continuous variables, and $\mathrm{P}$-values $<0.05$ were considered to indicate statistical significance. For categorical variables, the Fisher exact test and the chi-square test were used. P-values $<0.05$ were considered to indicate statistical significance. Statistical analyses were performed using PASW Statistics ver. 18.0 (SPSS Inc., Chicago, IL, USA). 


\section{RESULTS}

\section{Part 1}

A total of 29 KC bladder biopsies (17 males, 12 females) and 10 normal control biopsies were analyzed in the first part of this study. The urothelial expression of markers of apoptosis, mast cells, E-cadherin, ZO-1, and TGF- $\beta$ in KC and control biopsy specimens are shown in Table 1. The immunohistochemical staining of these specimens revealed significant differences between the $\mathrm{KC}$ and normal control bladder specimens in the TUNEL assay, mast cells, E- cadherin, and ZO-1 (Table 1, Fig.

Table 1. Urothelial expressions of apoptosis, mast cell acitivity, E-cadherin, ZO-1, and TGF- $\beta$ in biopsy specimens of ketamine related cystitis and normal control

\begin{tabular}{lccr}
\hline Variable & $\begin{array}{c}\text { Normal } \\
(\mathrm{n}=10)\end{array}$ & $\begin{array}{c}\text { Ketamine cystitis } \\
(\mathrm{n}=29)\end{array}$ & P-value \\
\hline Age $(\mathrm{yr})$ & $50.5 \pm 9.6$ & $25.4 \pm 4.3$ & \\
Sex, male:female & $0: 10$ & $17: 12$ & \\
TUNEL & $0.08 \pm 0.26$ & $3.63 \pm 2.38$ & $<0.001$ \\
Mast cell & $1.25 \pm 1.15$ & $8.75 \pm 5.69$ & $<0.001$ \\
E-cadherin & $42.41 \pm 16.73$ & $13.86 \pm 15.74$ & $<0.001$ \\
ZO-1 & $11.02 \pm 5.66$ & $3.64 \pm 4.77$ & $<0.001$ \\
TGF- $\beta$ & $1.10 \pm 0.52$ & $1.5 \pm 0.67$ & 0.141 \\
\hline
\end{tabular}

Values are presented as mean \pm standard deviation or number. TUNEL, terminal deoxynucleotidyl transferase-mediated dUTP nick end -labeling; ZO-1, zonula occludens-1; TGF, transforming growth factor.

1). However, no significant difference in TGF- $\beta$ expression was observed between the KC biopsy specimens and the normal controls (Table 1).

\section{Part 2}

A total of $16 \mathrm{KC}$ bladder specimens (10 males, 6 females) and 3 normal bladder specimens were analyzed in the second part of this study. Table 2 shows levels of urothelial sensory protein expression in the KC and control specimens. The Western blotting of these specimens revealed significant differences in $\mathrm{M} 3$ and $\beta 3$-AR between the $\mathrm{KC}$ and control bladder biopsies (Table

Table 2. The urothelial sensory protein expression in Ketamine cystitis and normal control

\begin{tabular}{lccc}
\hline Variable & $\begin{array}{c}\text { Normal } \\
(\mathrm{n}=3)\end{array}$ & $\begin{array}{c}\text { Ketamine cystitis } \\
(\mathrm{n}=16)\end{array}$ & P-value \\
\hline Age $(\mathrm{yr})$ & $54.7 \pm 10.5$ & $29.4 \pm 5.0$ & \\
Sex, male:female & $3: 0$ & $10: 6$ & \\
M2 & $0.12 \pm 0.21$ & $0.18 \pm 0.21$ & 0.314 \\
M3 & $0.14 \pm 0.93$ & $0.80 \pm 0.46$ & 0.034 \\
eNOS & $0.09 \pm 0.13$ & $0.07 \pm 0.09$ & 0.576 \\
iNOS & $0.02 \pm 0.23$ & $0.05 \pm 0.07$ & 0.737 \\
$\beta 3-A R$ & $1.05 \pm 0.13$ & $2.40 \pm 1.78$ & 0.034 \\
P2X & $1.30 \pm 0.16$ & $1.42 \pm 0.78$ & 0.911 \\
\hline
\end{tabular}

Values are presented as mean \pm standard deviation or number. eNOS, endothelial nitric oxide synthase; iNOS, inducible nitric oxide synthase; $\beta 3-A R, \beta-3$ adrenergic receptor.
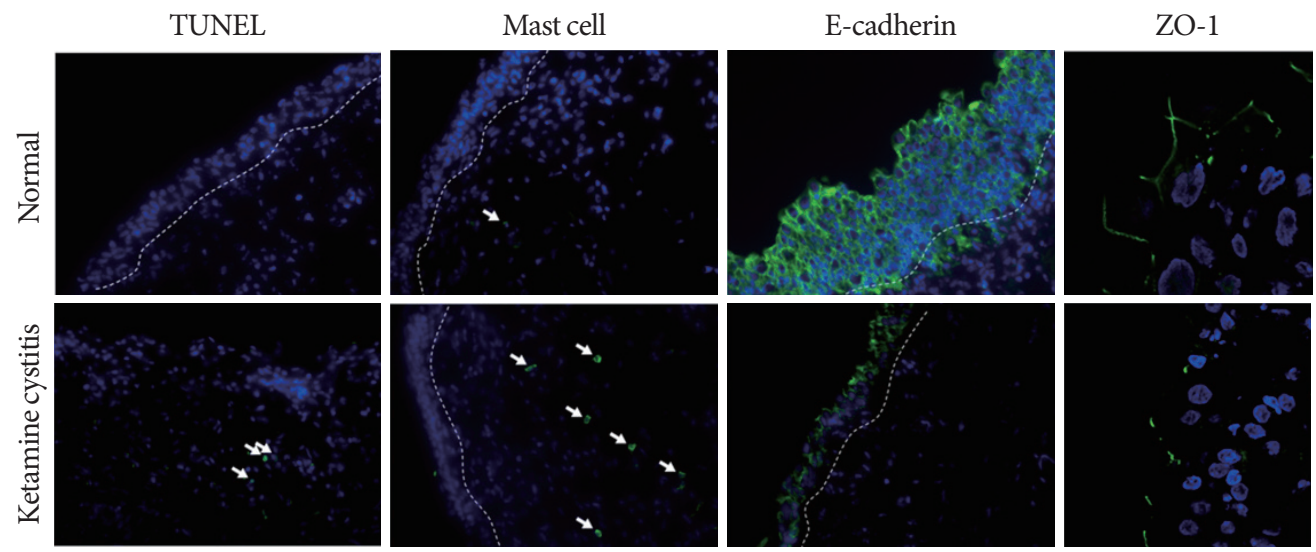

Fig. 1. Representative images from a biopsy taken from a patient with ketamine-related cystitis (KC) versus a control noninflamed bladder (immunohistochemistry, $\times 400$ ). For terminal deoxynucleotidyl transferase-mediated dUTP nick end-labeling (TUNEL), the target cells are labeled in green (arrows) and the white dotted line indicates the boundary of the urothelium; for mast cells, the target cells are labeled in green (arrows) and the white dotted line indicates the boundary of the urothelium; for E-cadherin, the target adhesion glycoprotein is labeled in green and the white dotted line indicates the boundary of the urothelium; for zonula occludens-1 (ZO1), the target junction protein is labeled in green. The sample size was $n=10$ for the normal samples and $n=29$ for the KC samples. 
2). No significant differences in expression were observed in the other sensory proteins that were evaluated.

\section{DISCUSSION}

The exact etiopathological mechanism underlying the development of severe bladder dysfunction and chronic bladder pain with ketamine use remains unknown. Though a number of mediators may be involved in the symptoms in question, it is likely that $\mathrm{KC}$ is associated with the detrimental toxic effects of ketamine and its active metabolites on the bladder epithelium $[8,9]$. In our study, significant changes to urothelial structural and barrier proteins in biopsied bladder tissue samples from KC patients were identified. The TUNEL assay, which is indicative of urothelial apoptosis, revealed a higher expression of apoptotic markers among KC specimens than in the normal controls. The expression of E-cadherin, which is a calcium-dependent cell-cell adhesion glycoprotein, was significantly lower in KC specimens than in the normal controls. ZO-1, a human epithelium tight junction protein, was significantly less expressed in KC bladder tissue than in the normal controls. This evidence suggests that $\mathrm{KC}$ produces significant changes in the urothelium, which may leave the stroma exposed to the toxic metabolites of ketamine and in turn lead to inflammation and the resultant KC symptoms. This may suggest that the toxic effect of ketamine on urothelium is twofold: a direct toxic effect on the superficial cells is followed by a receptor-mediated effect that damages the deeper tissue. This might explain why chronic bladder pain conditions such as IC/BPS and KC share many of the same clinical and pathological features, but the degree of bladder wall inflammation and fibrosis appears to be more severe in KC.

Mast cell activation is likely to play a crucial role in the immune-mediated response underlying many bladder pain conditions. In the present study, KC tissue samples exhibited a greater quantity of mast cells than the normal controls. In addition, TGF- $\beta$, a mediator that plays a role in wound healing by regulating the proliferation of epidermal and dermal cells near the injured site, was also significantly elevated in the KC specimens. We suggest that ketamine and/or its active metabolites result in significant alterations in the urothelial barrier, resulting in prominent inflammation in the subepithelial layer. These findings also mirror the findings of our previous study supporting a negative association between the number of activated mast cells and maximal bladder capacity, as well as severe lower urinary tract symptoms, in patients with $\mathrm{KC}$ in contrast to those diagnosed with IC/BPS [10].

Although KC shares many similarities with IC/BPS in terms of patient symptoms, the severity of lower urinary tract symptoms in KC is greater than in IC/BPS. In addition, most effective treatments for IC/BPS have been found to be minimally or not at all helpful in the treatment of KC. A previous animal study revealed altered muscarinic receptor expression in cyclophosphamide-induced cystitis [11]. In that study, the expression of muscarinic receptors in the cyclophosphamide-treated rats was significantly greater both in the urothelium and smooth muscle. However, another recent study of a ketaminetreated animal model revealed increased P2X receptor expression in ketamine-treated mouse bladder, while $\mathrm{M} 2$ and $\mathrm{M} 3$ receptor expression remained unchanged [12]. Our study is the first to have assessed sensory protein expression in human patients diagnosed with KC. In this study, we detected a significant increase in the M3 muscarinic receptor subtype, but not in the M2 subtype. In contrast, we did not detect any significant differences in $\mathrm{P}_{2} \mathrm{X}_{3}$ receptor expression in KC patient biopsies. While a number of possible explanations exist, augmented urothelial M3 receptor expression in pathological analyses may be associated with an increased release of transmitters that are associated with contractility and urgency, and thus may play a role in the clinical picture of $\mathrm{KC}$. Interestingly, we also found increased $\beta 3$-AR expression in the KC urothelium. The role of urothelial $\beta 3$-AR on bladder function has not been fully explored, although it may potentially play a role in compensating for the dysregulation of sensory proteins and mediate the relaxation of detrusor contractions.

NOS consists of a family of enzymes catalyzing the production of nitric oxide from L-arginine, and are essential for the production of the cellular signaling molecule nitric oxide. The predominant NOS isoform in the vasculature is eNOS, which helps modulate vascular tone, angiogenesis, and neural development. In contrast, iNOS is a distinct isoform of NOS that is involved in modulating inflammatory and immune responses. The increased expression of these 2 NOS isoforms has been reported to occur in tissue samples obtained from animal models of KC $[5,12]$. However, in our study we did not observe any increase in either of the 2 NOS isoforms in bladder tissue. The overexpression of eNOS and iNOS in animals following chronic treatment with ketamine may be related to alterations in sensitivity to ketamine, differences in ketamine metabolism between humans and rodents, discontinuation or reductions in 
ketamine use among the KC patients enrolled in our study, or medication-induced NOS expression during the period of treatment. Therefore, the involvement of the NOS system in KC patients remains controversial.

The main limitation of the current retrospective study is the small and unequal number of cases in the patient groups. In addition, age-related changes in the expression of certain proteins may hinder comparisons between the older control tissue samples and the younger KC tissue samples. However, age-related changes in the human urinary bladder have rarely been explored, except for age-related changes in bladder neurotransmission [13]. The main reason for this is that such studies would involve invasive procedures, and the complexity of human aging studies is exacerbated by the presence of associated chronic diseases and drugs that may affect the urinary bladder. $\mathrm{KC}$ is a disease that specifically occurs in younger individuals who abuse ketamine as a recreational drug. It is difficult to obtain control bladder samples from healthy young people. Thus, a prospective multicenter study examining the influence of ketamine on the expression of sensory and structural targets more thoroughly in being planned for the future.

In conclusion, human $\mathrm{KC}$ is associated with severe urothelial dysfunction due to altered urothelial structural proteins and apoptosis, an increased quantity of mast cells, and elevated expression of the muscarinic receptor M3 and $\beta 3$-AR. The expression of $\beta 3$-AR may play a role in modulating the release of transmitters from the urothelial mucosa, which may have functional implications for KC.

\section{REFERENCES}

1. Lankenau SE, Sanders B. Patterns of ketamine use among young injection drug users. J Psychoactive Drugs 2007;39:21-9.

2. Middela S, Pearce I. Ketamine-induced vesicopathy: a literature review. Int J Clin Pract 2011;65:27-30.
3. Wei YB, Yang JR, Yin Z, Guo Q, Liang BL, Zhou KQ. Genitourinary toxicity of ketamine. Hong Kong Med J 2013;19:341-8.

4. Chu PS, Ma WK, Wong SC, Chu RW, Cheng CH, Wong S, et al. The destruction of the lower urinary tract by ketamine abuse: a new syndrome? BJU Int 2008;102:1616-22.

5. Chuang SM, Liu KM, Li YL, Jang MY, Lee HH, Wu WJ, et al. Dual involvements of cyclooxygenase and nitric oxide synthase expressions in ketamine-induced ulcerative cystitis in rat bladder. Neurourol Urodyn 2013;32:1137-43.

6. Lin HC, Lee HS, Chiueh TS, Lin YC, Lin HA, Lin YC, et al. Histopathological assessment of inflammation and expression of inflammatory markers in patients with ketamine-induced cystitis. Mol Med Rep 2015;11:2421-8.

7. Chung SD, Wang CC, Kuo HC. Augmentation enterocystoplasty is effective in relieving refractory ketamine-related bladder pain. Neurourol Urodyn 2014;33:1207-11.

8. Tsai YC, Kuo HC. Ketamine cystitis: its urological impact and management. Urol Sci 2015;26:153-7.

9. Chen CH, Lee MH, Chen YC, Lin MF. Ketamine-snorting associated cystitis. J Formos Med Assoc 2011;110:787-91.

10. Lee CL, Jiang YH, Kuo HC. Increased apoptosis and suburothelial inflammation in patients with ketamine-related cystitis: a comparison with non-ulcerative interstitial cystitis and controls. BJU Int 2013;112:1156-62.

11. Giglio D, Ryberg AT, To K, Delbro DS, Tobin G. Altered muscarinic receptor subtype expression and functional responses in cyclophosphamide induced cystitis in rats. Auton Neurosci 2005;122:920.

12. Meng E, Chang HY, Chang SY, Sun GH, Yu DS, Cha TL. Involvement of purinergic neurotransmission in ketamine induced bladder dysfunction. J Urol 2011;186:1134-41.

13. Yoshida M, Homma Y, Inadome A, Yono M, Seshita H, Miyamoto Y, et al. Age-related changes in cholinergic and purinergic neurotransmission in human isolated bladder smooth muscles. Exp Gerontol 2001;36:99-109. 